\title{
A Review of Surface-Enhanced Raman Spectroscopy on Potential Clinical Applications Towards Diagnosing Colorectal Cancer
}

\author{
Owen Liang1, Ya-Hong Xie ${ }^{1,2, *}$ \\ ${ }^{1}$ Department of Materials Science and Engineering, University of California, \\ Los Angeles, Los Angeles, California, USA \\ ${ }^{2}$ Jonsson Comprehensive Cancer Center, University of California, Los Angeles, \\ Los Angeles, California, USA \\ *Corresponding author: Ya-Hong Xie, UCLA, HSSEAS School of Engineering \& Ap- \\ plied Sciences, Department of Materials Science and Engineering, 410 Westwood Pla- \\ za, 3121-F Engineering V, Los Angeles, CA 90095-1595, USA. Tel: +13108252971; \\ Fax:+13102067353;E-mail:yhx@ucla.edu
}

DOI: $10.30699 /$ mci.5.1.457-1

Submitted: 9 September 2020

Revised: 25 October 2020

Accepted: 15 November 2020

e-Published: 1 December 2020

\section{Keywords:}

Colorectal Neoplasms

Spectrum Analysis, Raman

Molecular Imaging

Machine Learning

Algorithms

Early Detection of Cancer $\dagger$ Both authors contributed equally to the manuscript.

Colorectal cancer (CRC) is one of the leading cancers in the world and early-screening is still the best method of cancer patient survival. However, colonoscopy as the current gold standard is not without flaws and an emerging technique called surface-enhanced Raman spectroscopy (SERS) coupled with machine learning is a possible candidate that could be applied in parallel with colonoscopy. This paper looks into the principles of SERS along with one of the most used machine learning algorithms: principal component analysis (PCA), and linear discriminate analysis (LDA). Case studies will be presented in the SERS application towards early screening, targeted imaging, and alternative diagnosis. The paper will conclude with the authors' analysis of the current landscape of SERS implementation into clinical applications. This review article highlights the promising technology of SERS as a potentially useful tool for clinicians and calls their attention toward this emerging technology.

\section{INTRODUCTION}

Colorectal cancer (CRC) is one of the leading cancers in the world and it is one of the top 5 leading in incidence, mortality, and societal cost. Taking the US as an example, colorectal is the 2nd most diagnosed cancer. A study in the US from 2012-2016, showed that the incidence was 381,000 , with 131,800 mortalities, and 649,000 survivals based on the 5-year relative survival rate [1]. The worldwide statistics showed
CRC as the third most common cancer with an approximate annual incidence of 1.8 million new cases and an annual mortality of over 800,000 cases $[2,3]$. In terms of societal cost, the national cost in the US was around 14 billion dollars for cancer care in 2010 and is expected to grow to 17 billion dollars in 2020 [4]. While improvements have been made in recent years due to early screening techniques, the typical gold standard 
of diagnosis is still endoscopy or colonoscopy [5]. Even in this respect, colonoscopy is not a guaranteed technique with a sensitivity of 75$93 \%$ for adenomas $\geq 6 \mathrm{~mm}$ in size [6]. The main difficulty lies in the polyps differentiation and the difficulty in identifying cancerous from benign growths. Also, on the patient side, colonoscopy is uncomfortable and potentially embarrassing so that it is usually not performed until the age of 50 , leading to missed diagnoses in the younger ages. Another example of an area of difficulty is ulcerative colitis which forms microscopic dysplasia in areas of inflammation and these are not visible with conventional colonoscopy. Raman spectroscopy (RS) is an emerging alternative technique; used in conjunction with specialized fiber-optic probes, it can provide a rapid and objective diagnosis of dysplastic areas thereby promoting tissue biopsy and/or polyp resection [7-10].

In this article, RS and surface-enhanced Raman spectroscopy (SERS) will be reviewed in the context of their application to the CRC diagnosis. For the sake of maintaining focus though, this review will be primarily limited to SERS with RS being explained as the background foundation. The first section begins with SERS application in early screening techniques without the need for imaging. Next, the focus will be shifted to imagining and how SERS could simultaneously be used with the current gold standard of endoscopy/colonoscopy for targeted imaging. Afterward, the next section will focus on the objective targeted diagnosis capability of SERS and how it could identify the tumor sites directly. Finally, the last section will expand upon the difficulties that SERS face in clinical adoption. While neither SERS nor RS has been approved for clinical use, it is the authors' objective to elucidate such a promising technology to the interests of clinicians with the hope that it will enter the clinics in near future.

\section{Principle of Raman Spectroscopy}

RS is a subset of vibrational spectroscopy. The general idea is that atomic bonds stretch, compress, and twist (generally termed as vibrational movements) at specific energies and these energies are specific to each type of molecules so that they can be used to identify specific bonds of a material. The combination of all these bond energies will give a fingerprint and studies have already shown the unique signatures of amino acids to peptides and the complex protein structures $[7,11]$. These two studies have a good compilation of the 20 most common amino acids with Raman peak locations corresponding to each bond vibration listed in tables and example fingerprint figures [12, 13]. Scattered photons are special phenomena in quantum physics where the process can be an inelastic collision, meaning the energy of the scattered photon is different from that of the incident photon by an amount equal to the vibrational energy. This inelastic collision can cause the emitted Raman photon to be of higher energy, anti-Stokes, lower energy, or Stokes shift. This inelastic collision is conceptualized as scattering, i.e. an instantaneous process and is fundamentally different from slower processes such as fluorescence, in which an atom/molecule is excited to a higher energy state and stay at the excited state for a limited time before decaying back to the ground state. Typically, Raman scattering is seen as if the molecule is put into a virtual state before almost instantaneously falling back into the ground state. The fundamental difference between Raman scattering and fluorescence is that photon absorption and subsequent re-emission are two sequential processes in fluorescence. However, Raman scattering is an instantaneous process/ collision between three particles, the incident photon, the atomic bond vibration quanta defined as a photon, and the scattered photon. This Raman scattering happens with an extremely low probability. Such interactions happen with 1 in a million probability. A laser source is necessary and for biological applications, SERS is necessary for practical applications (see next sub-section for details). The effective spectroscopic output is the intensity of the Raman scattering represented by the number of photons that the detector receives and is proportional to the molecular dipolepolarizability, and the energy axes which is the amount of absorbed energy that will cause the rotational and vibrational changes of the molecule $[7,11]$. In summary, RS provides exceedingly high levels of specificity; originating from the Raman active bonds, i.e. the basic molecular construction of the entity being measured. 


\section{Principle of Surface-Enhanced Raman Spec- troscopy}

SERS is a subset of the conventional RS. SERS occurs when a nanostructured surface is created to act as an antenna to induce a resonance between the surface plasmon of the metallic surface and the incoming photons; this creates an enhancement of the signal. A surface plasmon is the particle form of an oscillating electron cloud and through this photon-plasmon coupling, the vibration phonon modes are effectively enhanced. SERS is necessary because the scattering crosssection of the conventional Raman process is very weak, approximately one in every million to 100 million scattered photons, which is around 12-14 orders of magnitude lower than the fluorescence process [7]. Thus for biological applications, the RS signal is very difficult to be differentiated from the noise baseline unless a long exposure time is performed at every measurement spot. For biological applications of SERS, silver and gold are mostly applied as the metallic substrate with silver giving a better enhancement factor and gold being biologically compatible. In addition, both materials have their SERS resonance frequency in the visible light range and thus can be easily excited by conventional laser sources to give orders of magnitude enhancement [14]. Figure 1A shows a schematic of how SERS measurement might take place. In summary, the fundamental difference between conventional Raman and SERS (Raman+plasmon resonance) is the signal intensity enhancement, i.e. SERS makes RS practical.

\section{Data Analysis}

In analyzing the SERS dataset, typically studies rely on multivariate analysis tools and/or machine learning algorithms. The depth and complexity of either method are review articles on their own, so the main focus for this review will be on the principal component analysis (PCA) and linear discriminant analysis (LDA), as they are the most widely used methods. PCA is a dimension reduction method and measures the variance of a dataset. PCA can transform a dataset with many variables (i.e. 30 variables from 30 selected peaks of a SERS spectrum) to a 2D graph plotted by its two principal components. This process gives a more easily

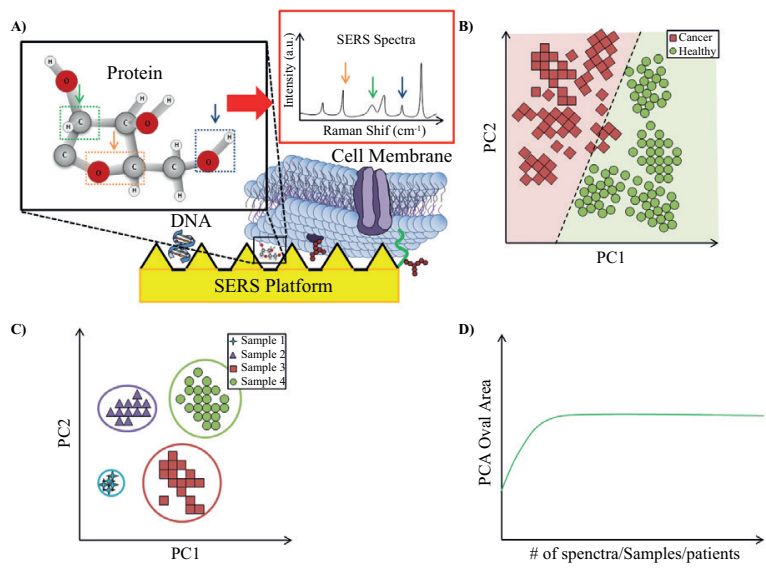

Figure 1: Schematic of the Surface-Enhanced Raman Spectroscopy Measurement and Analysis

A) SERS measurements can be done on a protein molecule with specific bonds of its amino acids, as shown in the insert black box. Examples of those bonds are represented by their respective colors: green C-H, orange C-O, and blue O-H. Each of these bonds will have a unique SERS peak as shown on the insert red box; representing a possible SERS spectrum. Other examples include nucleic acids and the surface proteome of a cell membrane; B) shows the subsequent analysis done through PCA-LDA. The complex SERS spectra are re-represented into the PCA space by their respective cancer and healthy datasets. A classifier algorithm such as LDA is performed on the PCA to give their respective classifying labels, in this case, red is cancerous and green is a healthy sample. Sensitivity and specificity can be calculated from the data points that fall into the correct/incorrect categories; in this example, the cancerous red square is misclassified into the healthy green region, termed a false negative, therefore the sensitivity is less than $100 \%$ as opposed to the $100 \%$ specificity; C) PCA shows the inherent variance amongst its data and if an oval encompasses each dataset, the area of that oval can represent the biovariability. This inherent biovariability will be saturated if enough spectra are taken per sample, or enough patients are measured per disease; D) a line graph can be used to represent such a phenomenon where saturation is the biovariability of a target disease and also explains the minimum amount of necessary patients for a study.

digestible visualization and is more simply analyzed from a human perspective (our minds work bettervisualizing data in $2 \mathrm{D}$ or $3 \mathrm{D}$ spaces than higher dimensions). PCA can be thought of as an orthogonal transformation to accentuate the differences and commonalities across datasets within the metric of variances [15]. Figure 1B shows a possible PCA graph from a SERS measurement. To differentiate between cancerous and healthy biomarkers, a machine learning algorithm is usually applied and LDA is one of the most prominent algorithms. LDA is a supervised machine learning algorithm meaning that the algorithm requires a known/labeled dataset to train its internal model. The algorithm works by applying a proportional weight constant to a variable 
in a linear function to output a classifier score. The variables, in this case, are the SERS feature peaks. The LDA algorithm can then split the PCA graph into the two classifying categories and thus a specificity and sensitivity value can be calculated [16]. In regards to clinical applicability, disease diagnosis is heavily dependent on the patient's biovariability. This variability is based on the inherent patient's characteristics and there is always a question as to whether the uniqueness of a SERS fingerprint is greater than the patient's biovariability. The SERS nature of the biovariability can be attributed to the various intricacies of proteins, e.g. size, molecular composition, SERS polarizability, etc. In the SERS and PCA-LDA disease diagnosis scheme, hundreds of SERS spectra are measured per patient or many patients per disease category. Therefore the PCA representation can be depicted by ovals encompassing each dataset and the natural spread of the oval area can be thought of as the inherent biological variability, as seen in Figure 1C. To the best of our knowledge, biovariability is not an issue thus we believe the variations within SERS are greater than the biovariability found in patients: making clinical diagnosis possible [17]. Such a phenomenon will also mean this biovariability saturates in a line graph as seen in Figure 1D. Doing such a study will give the necessary information about the minimum number of measurements needed per patient to make an effective diagnosis. In summary, the minute differences in the extra high dimensional space of SERS can only be differentiated, grouped, and recognized by machine learning: a humanly impossible task.

\section{SERS Application for Early Screening}

For early screening of colorectal cancer, there are several gold-standard approaches. For the most part, colonoscopy is the gold standard to catch the early signs of cancer. One category of improvement from SERS is regarding the early detection of biomarkers in the blood serum and plasma. The polypoid lesions are the most common cell type to search for in colonoscopic evaluation, but if adenomatous polyps are found, they should be immediately removed as they are known to be pre-malignant [7]. Excisional tissue biopsies remain the definite cancer diagnosis despite their invasive drawbacks, such as internal bleeding, visceral perforation, and unnecessary removal of healthy tissue. One downside of colonoscopy is the large cost and great inconvenience to the patient. Examples of costs include the hiring of a skilled physician and the long procedure time; while examples of inconvenience to the patient include whole bowel cleansing with laxatives. Excisional biopsies remain the best standard for cancer diagnosis, but this method is invasive and impractical for early and large cohort screening. By just using conventional CRC early screening tools, the effectiveness of identifying early neoplasia and subtle lesions depends on the physician's skill, knowledge, experience, and many other factors [2]. The general approach for SERS detection of blood serum and plasma is to compare the cancerous patient's serum with that of a healthy control serum [7]. An example of this can be seen in the study of Lin et al., where SERS was done to differentiate the cancerous and healthy serum [2]. The study used gold nanoparticles and the $785 \mathrm{~nm}$ laser to differentiate patients with CRC compared with healthy controls from blood serum. Previous studies used silver nanoparticles but this particular study used gold nanoparticles. The study was able to show key Raman peaks to differentiate between normal and cancer serum. By applying statistical analysis such as principal component analysis and linear discriminate analysis, they were able to segregate the two types of samples with a sensitivity of $92.1 \%$ and specificity of $95.6 \%$.

Another approach is looking at the blood plasma to differentiate the colorectal and adenomatous polyps. Silver nanoparticles were used as the SERS probe in this case. The blood of 21 colorectal patients and 25 healthy individuals were extracted and incubated together. Using partial least square discriminate analysis, normal, cancer with polyps, and cancer without polyps samples were differentiated with a sensitivity of $86.4 \%$ and specificity of $80.0 \%$. This also translates to a receiver operating characteristics area under the curve (ROC AUC) of 0.869 to 0.945; this shows great promise in using SERS for this detection [18]. In addition, a follow-up study showed improvements that the SERS probe could be done using polarization. With the addition of polarization, the silver nanoparticles mixed with serum, the accuracy had improved to $91.6 \%$ or $89.5 \%$ sensitivity and $93.3 \%$ specificity [19]. Besides polyp measurement, other serum constituents can 
be measured with SERS. This study used SERS for the detection of albumin and globulin and measured the serum proteins. Typically, serum albumin is used to assess the nutritional status, the severity of the disease, disease progression, and prognosis. Therefore, measuring serum albumin is a good biomarker protein candidate for SERS. Coupled SERS and PCA-LDA was able to show a $100 \%$ separation between normal and CRC [20]. A separate group also did a similar study but focused on cancerrelated peptides for early screening. These peptides included tumor-specific antigen (TSA), tumorassociated antigens (TAA), and immunoglobulin, in this case, was silver nanohexagons [21].

Besides proteomic approaches, SERS can also assist in the genomic diagnosis of cancer. In particular, SERS can measure the conformational change of proteins and DNA $[22,23]$. The ability to measure the conformational change in the DNA can help correlate biomarkers for a high incidence of colorectal cancer. In particular, the study of MorlaFolch et al., shows that the K-Ras gene is correlated with the oncogenic response to colorectal cancer [23]. The configuration and shape of the gene can be measured with SERS and partial least-squares discriminate analysis was applied to differentiate the point mutations in the DNA fragments. Another emerging area of interest for early CRC screening is the usage of exosomes as an early biomarker. Exosomes are extracellular vesicles of $30-140 \mathrm{~nm}$ that contain molecular cargo, i.e. nucleic acids and proteins, with very efficient delivery from the original cell to a target cell. In addition, studies have already shown that cancerous cells release more exosomes than healthy cells so exosomes make a good candidate for a potential new biomarker as well as the convenient patient sample extraction, i.e. blood, saliva, and cerebral spinal fluid [24]. In regards to $\mathrm{CRC}$, several studies have already shown the presence of CRC cancer-specific proteins through exosomal secretion, such as cadherin-17 and EpCAM [25, 26]. While typically exosomal studies are done through proteomic techniques, SERS can also be used to analyze exosomes. One advantage of using SERS is the ability to analyze exosome contents individually as opposed to mass spectrometry in which tens of thousands of cells are required in the typical mass. Using PCA, exosomes from different parent sources were differentiated with clear sensitivity and specificity $[27,28]$. While there is currently a gap in knowledge for the specific application to CRC, multiple studies have shown the capability of SERS with exosomes [29-33].

\section{SERS Application for Targeted Imaging}

Colonoscopy or endoscopy is the gold standard for CRC diagnosis. This involves an optical camera and an optical fiber and trained personnel identifying abnormal polyp formation. However, such identification is not the best and can lead to inaccuracies. The SERS technology brings a new subset of colonoscopy by introducing targeted imaging with biomarkers to objectively select the correct polyp. Similar to the current endoscope, an optical fiber is used; however, at the tip of the fiber, SERS NPs are introduced to identify cancerous cells from a spectroscopic approach [34]. An example of this can be seen in the study of Garai et al., where a custom in-house prototype device was created to allow for in vivo studies of simulated colon organs [35]. This device resembles an endoscope but a mirror and a rotating camera setup are placed at the top of the device; this allows for a $360^{\circ}$ view of the simulated colon. A focusing lens onto an optical fiber end, which is coated with gold nanoparticles, is also applied for SERS measurement. They showed the effectiveness of the SERS imaging capabilities with different material compositions and thus diverse SERS signals were made that could easily differentiate various materials. In vivo studies were also performed on a pig's colon with a targeted cancer area proving the viability of the platform, before finally doing the 1 st human clinical trial on a routine colonoscopy of a male patient. Unfortunately, targeted fluorophores were not allowed to be applied without FDA approval so the 1st human trial was just to show the feasibility of collecting images from their custom prototype SERS-coated endoscope [35].

In another study, a beveled optical-fiber conical Raman probe was developed to study the epithelial tissue of patients with a high risk of cancer. Specifically, dysplasia was the focus of the study as it is a pre-cancer indicator and typically involves the epithelial tissue layer. Their invention of using a ball lens to focus the laser rays increased the signal by 19 times compared to a typical conical Raman probe. The group differentiated epithelial tissue in 
vivo to prove its concept and clinical applicability [36]. Finally, this study looked into the classification of neoplastic lesions, which can remove a large number of false positives, and also focuses on in vivo classifications. For most of the studies with Raman and SERS, the typical detection range is within the low-frequency ranges of $0-1800 \mathrm{~cm}-1$, otherwise known as the protein-rich region. This study focused on comparing this low-frequency regime along with the high-frequency regime of 2050-3100 cm-1, where typically the lipid peaks appear around $2900 \mathrm{~cm}-1$. The high-frequency regime showed fluorescence about half as much as the low-frequency regime. Although the highfrequency regime shows fewer characteristic peaks, the multivariate analyses showed this regime was better at predicting the pathology of cancer than the low-frequency spectra. Thus, they showed that Raman had great potential in improving in vivo detection of early neoplastic lesions for colorectal cancer [37].

\section{SERS Application for Alternative Diagnosis} Via labels

Besides the typical improvements to the optical imaging of colonoscopy, there are alternative diagnosis methods that can be done with Raman and SERS. These studies typically involve nanoparticles and conjugating the surface with antibodies or antigens for targeted therapy or diagnosis [7, 34]. The first in-depth example uses the antibody carcinoembryonic antigen (CEA) as the biomarker for detecting CRC in patients' serum. However, while CEA is typically expressed in most CRC patients, it is undetectable in normal colonic epithelial cells. Furthermore, it is found that CEA is also expressed in gastric, liver, and lung cancer. SERS is used to detect CEA by first binding anti-CEA onto gold/ silver core-shell nanoparticles and then using the platform like a probe for quantitative detection of CEA in serum. To correlate the CEA concentration with the SERS probe, a calibration curve was established and compared with electrochemical luminescence. SERS probe was applied to 26 patients' serum and the detection limit of $5 \mathrm{pg} / \mathrm{ml}$ of CEA was established. This technique establishes the feasibility of using the SERS probe as a clinical tool [38]. A similar study using the CEA as a biomarker has also been done, but with the twist of using a biocompatible magnetic fluid combined with a surface decorated maghemite nanoparticle. Two cell lines were used to test this SERS probe where one was highly expressing CEA (LS174T) and the other expressed low levels of CEA (HCT116). Electron micrographs showed preferential maghemite nanoparticle uptake by CEA-expressing tumor cells and additional evidence for the specificity of the CEA-expression tumor cells was proved with ELISA and Prussian blue iron staining. Their data suggested promising results of applying a drug delivery system for targeted therapy [39]. The study of Conde et al., looked into functionalizing gold nanoparticles for specific targeting of cancer biomarkers and coating with an antibody-drug conjugate for targeted FDAapproved drugs [40]. One such drug is Cetuximab, which is a monoclonal antibody that specifically targets the epidermal growth factor receptor (EGFR). Mice models were used to test the in vitro and in vivo nanoparticle tumor-specific targets and drug efficacy. Tumor size reduction provided further potentials of the clinical application of this SERS probe and drug delivery system [40].

\section{Label-free}

The alternative to a biomarker-based measurement is a label-free diagnosis and this is accomplished by looking at the overall spectrum of the cell or tissue and analyzing their cellular components [41-43]. An example of this is in the study of Lin et al., which looks into label-free detection of CRC in serum samples via SERS [2]. In particular, the increase/ decrease of relative amounts of nucleic acid, saccharide, lipids, and proteins will have a net effect on the overall spectrum. Multivariate statistical techniques such as PCA and LDA are applied to develop effective diagnostic algorithms for the classification of normal and cancerous serum. In this particular study, the accomplished accuracy was $97.4 \%$ with a ROC curve of 0.896 ; demonstrating the clinical applicability of the PCA-LDA-based diagnostic platform [2]. Another potential field for SERS is through the detection of circulating tumor cells (CTCs). The basic methodology behind this is to isolate tumor cells from the patients' blood and measure the Raman/SERS signature of the CTC. This will bypass the need for probing or biopsy of the cancerous tissue; however, the challenge comes from the CTC concentration for the patient's cancer 


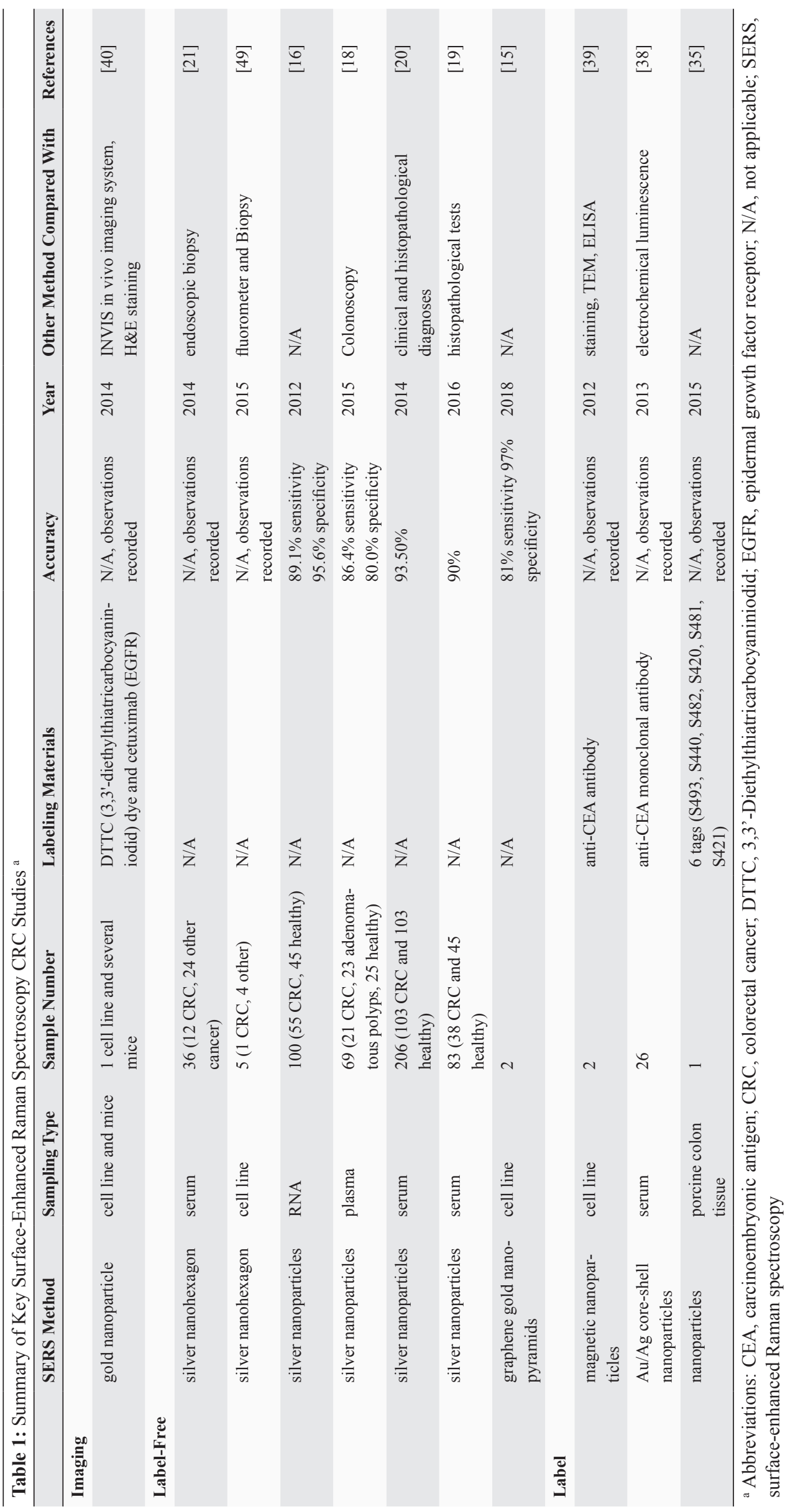


stage. To the best of our knowledge, there does not seem to be a well-established study of diagnosing CTC in CRC patients via SERS. Most of the literature contains proof-of-concept literature with cell differentiation from various blood cells, blood spiked with cell culture cells, and other types of cancer cells [44-47]. This is still an active field of research with much potential of utilizing the nondestructive quality of SERS to study the metastasis properties of CTCs [48]. Besides looking for CTCs in the patient blood, cell-free nucleic acids and epigenetics are also emerging fields where SERS has some potential. In the study of Ito et al., SERS of cell-free DNA (cfDNA) for CRC was explored from lysed cells obtained from cell culture and clinical serum [49]. The focus of this study was to highlight the high sensitivity of SERS for single-molecule detection in minute changes of a SERS peak due to particular DNA methylation. Other instances of using SERS for DNA methylation can also be found in these studies [50-52]. The specifics of the SERS detection of DNA epigenetic is focusing on the vibrational assignment of the cytosine and guanine nucleotides, where ring breathing, $\mathrm{CH} /$ $\mathrm{CH} 2$ deformation, and $\mathrm{C}=\mathrm{O}$ stretching modes are picked up with different intensities. In addition, the redshifting of vibrational modes can also indicate specific modifications [52].

\section{Cancer Cell States}

Another benefit of a SERS platform is the ability to differentiate various metabolic states of a cell. The study of Liang et al., shows the ability of a SERS probe in differentiating the surface proteome of living, burst, and dead (intact) states of CRC cell lines [15]. This is done through a nano-patterned substrate and differentiating the three types of SERS spectra without the use of a biomarker. PCA was the multivariate tool used along with a perception algorithm to differentiate the three different metabolic states with $89 \%$ accuracy [15]. A summary of the key articles mentioned in the previous sections is provided in Table 1.

\section{Realistic Difficulties of SERS Adoption to Clinics Credibility of SERS}

While the main portion of this review highlights the amazing potential of SERS as an emerging technology, the typical question about these types of review articles needs to be acknowledged: given so many encouraging results, why has SERS not yet been implemented in clinics/hospitals? Below we share our beliefs about this in two parts: 1) the typically small patient cohort size leading to insufficient validation in reproducibility and rigor for clinical implementation; 2) lack of correlation between SERS spectra signature to specific protein expression pathways. The former is rooted in biostatistics and requires clinical trials with large scale funding, and the latter represents pending (or missing) research that could potentially be associated with fundamental biological challenges yet to be met. For the first reason, the reproducibility of the abovementioned SERS studies for clinical applications is a big question mark in the field. Usually, a statistically relevant clinical study involves around 40 patients per measurable group depending on the disease type. To date, we have not come across a substantial amount of SERS clinical studies with this sort of severity [53,54]. An example of this can be seen in the previously mentioned study where only 12 healthy patients were measured compared to 12 patients with CRC. Of those 12 cancer patients, 7 were male and 5 were female with 3,7 , and 2 patients split between cancer stage II, stage III, and stage IV, respectively [21]. Such patient cohort size is far too small to make a distinguishable clinical case, but it is enough to present the technology as a feasible possible modality in the future. As mentioned before, the translation of technology from the laboratory to clinics does require consorted efforts often organized by federal agencies such as the US National Institutes of Health.

In the second explanation, all SERS studies we have come across tackle just diagnosis of the clinical disease without relating to the possible mechanism. Although SERS is touted as a proteomics technique, there are still technical difficulties correlating the observed SERS peaks with protein expression pathways. A good proteomic technique for comparison is with mass spectrometry where individual proteins are broken down to peptides and the subsequent amino acid sequence can be analyzed to form a conclusion with the corresponding gene and protein expression pathway. The working principle of SERS, on the other hand, dictates that the spectral peak heights are directly correlated to the abundance of one or more types of amino acids. With individual proteins being composed of numerous amino acids, it remains an unanswered 
question as to whether the information about the abundance of amino acids could allow for unique connections to the abundance of proteins. This is in our opinion a fundamental challenge confronting SERS. It should be stressed here that even if such a direct correlation with proteome is impossible, it does not diminish the potential of SERS being used as a "molecular fingerprint", in other words clinically worthy biomarkers for disease diagnosis. However, the path to its acceptance into clinical practice will be expected to be much more unfaithful because it is lacking a clear proteomic or genomic foundation.

\section{Comparison to Other Methods}

Besides the acceptability of SERS as a proteomic technique, there is also the consideration of comparing SERS to other established CRC diagnostic methods. While the comparison of the advantages and disadvantages for all CRC diagnostic techniques is beyond the scope of this review, one of the generally agreed modalities in CRC diagnostic are fecal occult blood test (FOBT) or fecal immunochemical test (FIT) [55-58], next-generation sequencing (NGS) $[59,60]$, and colonoscopy as the gold standard technique [61]. From these, colonoscopy has been already compared with improvements in imaging, using SERS as mentioned in the previous sections. For FOBT and FIT, patients' stool needs to be collected and sensitive assays for the detection of blood within the stool sample are already wellestablished; so that there is no need for SERS. There is an alternative genetic diagnostic method called Cologuard $\AA$ already on the market; this solidifies the lack of a stool sample diagnostic method [56]. The best area that SERS can complement is NGS techniques in the area of liquid biopsies, as it is considered the holy grail of cancer diagnosis. Here is the basic concept: a patient going in for a routine blood draw can be used to identify biomarkers that pinpoint an early CRC diagnosis. For cfDNA, a recent study has shown the capability of this method with NGS technology [59]. It is unknown if SERS is better or worse than NGS as this is an active field of research, but SERS can complement by measuring proteomic biomarkers instead of genomic biomarkers. In addition, the single molecular sensitivity of SERS allows it to handle smaller biomarker concentrations leading to the possibility of earlier stage diagnosis, where early cancer stages lack an abundance of differentiable biomarkers. For a liquid biopsy, the cost-benefit of NGS and SERS, when compared to colonoscopy, is superb as routine blood work can offset the expense and discomfort of colonoscopy. NGS is more easily adaptable to the current infrastructure when compared with SERS so capital cost-wise, it is more favorable. However, if label-free SERS is implemented, there are no reagent costs and the fabrication techniques of SERS make each assay extremely cheap per patient. The initial costs to implement SERS are the spectrometer and the database formation which requires validation of a randomized control clinical trial; all of which makes NGS the currently cost-benefit technique.

\section{CONCLUSION}

$\mathrm{CRC}$ is one of the leading cancers in the world and most innovations focus on early screening and early detection for successful prevention of the disease. SERS is not different and aims to complement the colonoscopy as the gold standard technique for early detection through serum, targeted optical imagining, and targeted diagnosis and therapy. The individual constituents such as peptides, proteins, lipids, nucleic acids, saccharides, and extracellular vesicles can all be measured in blood serum and plasma with SERS and subsequently used to differentiate healthy from CRC patients. In targeted imaging applications, nanoparticles are coated onto fiberoptic probes of endoscopes and this subsequently allows for quantitative diagnosis of polyps based on SERS signals rather than qualitative image analysis by expert clinicians. For targeted diagnosis, nanoparticles can also be conjugated with antibodies or antigens to specifically target CRC sites. Positive measurements with SERS can then subsequently allow targeted treatment as these nanoparticles can also be conjugated with current FDA approved medications. The objective of this review article was to present the many aspects of SERS as the next generation diagnostic technique for clinical applications in patients with CRC.

\section{ACKNOWLEDGMENTS}

YHX acknowledges the support of the Alexander von Humboldt Foundation. This work was funded in part by a grant from the United States Government, administered by the National Academy of Sciences (Grant No. 200008303), and the generous support of the American people through the United States 
Department of State and the United States Agency for International Development (USAID) under the Pakistan - U.S. Science \& Technology Cooperation Program. The contents do not necessarily reflect the views of the United States Government.

\section{CONFLICT OF INTEREST}

The authors declared no conflict of interest.

\section{ETHICS APPROVAL}

Not Applicable.

\section{REFERENCES}

1. Howlader N, Noone AM, Krapcho M, Miller D, Brest A, Yu M, et al. SEER Cancer Statistics Review, 1975-2016, Bethesda, MD: National Cancer Institute; 2020 [updated 2020 April 9; cited 2020 April 9]. Available from: https:// seer.cancer.gov/archive/csr/1975 2016/.

2. Lin D, Feng S, Pan J, Chen Y, Lin J, Chen G, et al. Colorectal cancer detection by gold nanoparticle based surface-enhanced Raman spectroscopy of blood serum and statistical analysis. Opt Express. 2011;19(14):13565-77. DOI: $10.1364 /$ OE.19.013565 PMID: 21747512.

3. Globocan. Global Cancer Observatory: Colorectal Cancer. Lyon, France International Agency for Research on Cancer; 2019 [updated 2019 Feb; cited 2020 Nov 19]. Available from: https://gco.iarc.fr/today/data/factsheets/cancers/10 8 9-Colorectum-fact-sheet.pdf.

4. Mariotto AB, Yabroff KR, Shao Y, Feuer EJ, Brown ML. Projections of the cost of cancer care in the United States: 2010-2020. J Natl Cancer Inst. 2011;103(2):117-28. DOI: 10.1093/jnci/djq495 PMID: 21228314.

5. Moiel D, Thompson J. Early detection of colon cancer-the kaiser permanente northwest 30-year history: how do we measure success? Is it the test, the number of tests, the stage, or the percentage of screen-detected patients? Perm J. 2011;15(4):30-8. DOI: 10.7812/tpp/11-128 PMID: 22319413.

6. Lin JS, Piper MA, Perdue LA, Rutter CM, Webber EM, O'Connor E, et al. Screening for Colorectal Cancer: Updated Evidence Report and Systematic Review for the US Preventive Services Task Force. JAMA. 2016;315(23):257694. DOI: 10.1001/jama.2016.3332 PMID: 27305422.

7. Ravanshad R, Karimi Zadeh A, Amani AM, Mousavi SM, Hashemi SA, Savar Dashtaki A, et al. Application of nanoparticles in cancer detection by Raman scattering based techniques. Nano Rev Exp. 2018;9(1):1373551. DOI: 10.1080/20022727.2017.1373551 PMID: 30410710.

8. Shim MG, Song LM, Marcon NE, Wilson BC. In vivo near-infrared Raman spectroscopy: demonstration of feasibility during clinical gastrointestinal endoscopy. Photochem Photobiol. 2000;72(1):146-50. DOI: 10.1562/0031-8655(2000)072<0146:IVNIRS >2.0.CO;2 PMID: 10911740.

9. Molckovsky A, Song LM, Shim MG, Marcon NE, Wilson
BC. Diagnostic potential of near-infrared Raman spectroscopy in the colon: differentiating adenomatous from hyperplastic polyps. Gastrointest Endosc. 2003;57(3):396-402. DOI: $10.1067 / \mathrm{mge} .2003 .105$ PMID: 12612529 .

10. Bergholt MS, Zheng W, Ho KY, Teh M, Yeoh KG, So JB, et al. Fiber-optic Raman spectroscopy probes gastric carcinogenesis in vivo at endoscopy. J Biophotonics. 2013;6(1):4959. DOI: 10.1002/jbio.201200138 PMID: 23288709.

11. Kallaway C, Almond LM, Barr H, Wood J, Hutchings J, Kendall C, et al. Advances in the clinical application of Raman spectroscopy for cancer diagnostics. Photodiagnosis Photodyn Ther. 2013;10(3):207-19. DOI: 10.1016/j.pdpdt.2013.01.008 PMID: 23993846.

12. Jenkins AL, Larsen RA, Williams TB. Characterization of amino acids using Raman spectroscopy. Spectrochim Acta A Mol Biomol Spectrosc. 2005;61(7):1585-94. DOI: 10.1016/j.saa.2004.11.055 PMID: 15820892.

13. Zhu G, Zhu X, Fan Q, Wan X. Raman spectra of amino acids and their aqueous solutions. Spectrochim Acta A Mol Biomol Spectrosc. 2011;78(3):1187-95. DOI: 10.1016/j. saa.2010.12.079 PMID: 21242101.

14. Le Ru EC, Etchegoin PG. Chapter 2 - Raman spectroscopy and related optical techniques. In: Le Ru EC, Etchegoin PG, editors. Principles of Surface-Enhanced Raman Spectroscopy. Amsterdam: Elsevier; 2009. p. 29-120. DOI: 10.1016/B978-0-444-52779-0.00008-8.

15. Liang O, Wang P, Xia M, Augello C, Yang F, Niu G, et al. Label-free distinction between p53+/+ and p53 -/- colon cancer cells using a graphene based SERS platform. Biosens Bioelectron. 2018;118:108-14. DOI: 10.1016/j. bios.2018.07.038 PMID: 30059864.

16. Chen Y, Chen G, Feng S, Pan J, Zheng X, Su Y, et al. Label-free serum ribonucleic acid analysis for colorectal cancer detection by surface-enhanced Raman spectroscopy and multivariate analysis. J Biomed Opt. 2012;17(6):067003. DOI: 10.1117/1.JBO.17.6.067003 PMID: 22734781.

17. Mohammed D, Matts PJ, Hadgraft J, Lane ME. In vitro-in vivo correlation in skin permeation. Pharm Res. 2014;31(2):394-400. DOI: 10.1007/s11095-013-1169-2 PMID: 23943545.

18. Feng S, Wang W, Tai IT, Chen G, Chen R, Zeng H. Label-free surface-enhanced Raman spectroscopy for detection of colorectal cancer and precursor lesions using blood plasma. Biomed Opt Express. 2015;6(9):3494-502. DOI: 10.1364/BOE.6.003494 PMID: 26417518.

19. Lin D, Huang H, Qiu S, Feng S, Chen G, Chen R. Diagnostic potential of polarized surface enhanced Raman spectroscopy technology for colorectal cancer detection. Opt Express. 2016;24(3):2222-34. DOI: 10.1364/OE.24.002222 PMID: 26906798 .

20. Wang Z, Wei D, Wei L, He Y, Shi G, Wei X, et al. Aberration correction during real time in vivo imaging of bone marrow with sensorless adaptive optics confocal microscope. J Biomed Opt. 2014;19(8):086009. DOI: 10.1117/1. JBO.19.8.086009 PMID: 25117079.

21. Ito $H$, Inoue $H$, Hasegawa $K$, Hasegawa $Y$, Shimizu $T$, Kimura S, et al. Use of surface-enhanced Raman scattering 
for detection of cancer-related serum-constituents in gastrointestinal cancer patients. Nanomedicine. 2014;10(3):599608. DOI: 10.1016/j.nano.2013.09.006 PMID: 24103303.

22. Yu X, Hayden EY, Xia M, Liang O, Cheah L, Teplow DB, et al. Surface enhanced Raman spectroscopy distinguishes amyloid Beta-protein isoforms and conformational states. Protein Sci. 2018;27(8):1427-38. DOI: 10.1002/pro.3434 PMID: 29700868.

23. Morla-Folch J, Gisbert-Quilis P, Masetti M, Garcia-Rico E, Alvarez-Puebla RA, Guerrini L. Conformational SERS Classification of K-Ras Point Mutations for Cancer Diagnostics. Angew Chem Int Ed Engl. 2017;56(9):2381-5. DOI: 10.1002/anie.201611243 PMID: 28079954.

24. Tovar-Camargo OA, Toden S, Goel A. Exosomal microRNA Biomarkers: Emerging Frontiers in Colorectal and Other Human Cancers. Expert Rev Mol Diagn. 2016;16(5):55367. DOI: 10.1586/14737159.2016.1156535 PMID: 26892862.

25. Bernhard OK, Greening DW, Barnes TW, Ji H, Simpson RJ. Detection of cadherin-17 in human colon cancer LIM1215 cell secretome and tumour xenograft-derived interstitial fluid and plasma. Biochim Biophys Acta. 2013;1834(11):2372-9. DOI: 10.1016/j.bbapap.2013.03.022 PMID: 23557862.

26. Ostenfeld MS, Jensen SG, Jeppesen DK, Christensen LL, Thorsen SB, Stenvang J, et al. miRNA profiling of circulating $\operatorname{EpCAM}(+)$ extracellular vesicles: promising biomarkers of colorectal cancer. J Extracell Vesicles. 2016;5:31488. DOI: 10.3402/jev.v5.31488 PMID: 27576678.

27. Yan Z, Dutta S, Liu Z, Yu X, Mesgarzadeh N, Ji F, et al. A Label-Free Platform for Identification of Exosomes from Different Sources. ACS Sens. 2019;4(2):488-97. DOI: 10.1021/acssensors.8b01564 PMID: 30644736.

28. Galamb O, Bartak BK, Kalmar A, Nagy ZB, Szigeti KA, Tulassay Z, et al. Diagnostic and prognostic potential of tissue and circulating long non-coding RNAs in colorectal tumors. World J Gastroenterol. 2019;25(34):5026-48. DOI: 10.3748/wig.v25.i34.5026 PMID: 31558855.

29. Wang Z, Zong S, Wang Y, Li N, Li L, Lu J, et al. Screening and multiple detection of cancer exosomes using an SERSbased method. Nanoscale. 2018;10(19):9053-62. DOI: 10.1039/c7nr09162a PMID: 29718044.

30. Stremersch S, Marro M, Pinchasik BE, Baatsen P, Hendrix A, De Smedt SC, et al. Identification of Individual Exosome-Like Vesicles by Surface Enhanced Raman Spectroscopy. Small. 2016;12(24):3292-301. DOI: 10.1002/ smll.201600393 PMID: 27171437.

31. Park J, Hwang M, Choi B, Jeong H, Jung JH, Kim HK, et al. Exosome Classification by Pattern Analysis of Surface-Enhanced Raman Spectroscopy Data for Lung Cancer Diagnosis. Anal Chem. 2017;89(12):6695-701. DOI: 10.1021/acs.analchem.7b00911 PMID: 28541032.

32. Zong S, Wang L, Chen C, Lu J, Zhu D, Zhang Y, et al. Facile detection of tumor-derived exosomes using magnetic nanobeads and SERS nanoprobes. Anal Methods. 2016;8(25):5001-8. DOI: 10.1039/C6AY00406G.

33. Lee C, Carney RP, Hazari S, Smith ZJ, Knudson A, Robert- son CS, et al. 3D plasmonic nanobowl platform for the study of exosomes in solution. Nanoscale. 2015;7(20):9290-7. DOI: 10.1039/c5nr01333j PMID: 25939587.

34. Kim Y-i, Jeong S, Jun B-H, Lee Y-S, Lee Y-S, Jeong DH, et al. Endoscopic imaging using surface-enhanced Raman scattering. Eur J Nanomed. 2017;9(3-4):91. DOI: 10.1515/ ejnm-2017-0005.

35. Garai E, Sensarn S, Zavaleta CL, Loewke NO, Rogalla $\mathrm{S}$, Mandella MJ, et al. A real-time clinical endoscopic system for intraluminal, multiplexed imaging of surface-enhanced Raman scattering nanoparticles. PLoS One. 2015;10(4):e0123185. DOI: 10.1371/journal. pone.0123185 PMID: 25923788 .

36. Wang J, Bergholt MS, Zheng W, Huang Z. Development of a beveled fiber-optic confocal Raman probe for enhancing in vivo epithelial tissue Raman measurements at endoscopy. Opt Lett. 2013;38(13):2321-3. DOI: 10.1364/ OL.38.002321 PMID: 23811915.

37. Short MA, Tai IT, Owen D, Zeng H. Using high frequency Raman spectra for colonic neoplasia detection. Opt Express. 2013;21(4):5025-34. DOI: 10.1364/OE.21.005025 PMID: 23482035.

38. Chen G, Chen Y, Zheng X, He C, Lu J, Feng S, et al. Surface-enhanced Raman scattering study of carcinoembryonic antigen in serum from patients with colorectal cancers. Applied Physics B. 2013;113(4):597-602. DOI: 10.1007/ s00340-013-5515-1.

39. da Paz MC, Santos Mde F, Santos CM, da Silva SW, de Souza LB, Lima EC, et al. Anti-CEA loaded maghemite nanoparticles as a theragnostic device for colorectal cancer. Int J Nanomedicine. 2012;7:5271-82. DOI: 10.2147/IJN. S32139 PMID: 23055733.

40. Conde J, Bao C, Cui D, Baptista PV, Tian F. Antibody-drug gold nanoantennas with Raman spectroscopic fingerprints for in vivo tumour theranostics. J Control Release. 2014;183:87-93. DOI: 10.1016/j.jconrel.2014.03.045 PMID: 24704711.

41. Andrade PO, Bitar RA, Yassoyama K, Martinho H, Santo AM, Bruno PM, et al. Study of normal colorectal tissue by FT-Raman spectroscopy. Anal Bioanal Chem. 2007;387(5):1643-8. DOI: 10.1007/s00216-006-0819-1 PMID: 17031621.

42. Widjaja E, Zheng W, Huang Z. Classification of colonic tissues using near-infrared Raman spectroscopy and support vector machines. Int J Oncol. 2008;32(3):653-62. PMID: 18292943.

43. Scalfi-Happ C, Udart M, Hauser C, Rück A. Investigation of lipid bodies in a colon carcinoma cell line by confocal Raman microscopy. Med Laser Appl. 2011;26(4):152-7. DOI: 10.1016/j.mla.2011.08.002.

44. Sha MY, Xu H, Natan MJ, Cromer R. Surface-enhanced Raman scattering tags for rapid and homogeneous detection of circulating tumor cells in the presence of human whole blood. J Am Chem Soc. 2008;130(51):17214-5. DOI: $10.1021 / \mathrm{ja} 804494 \mathrm{~m}$ PMID: 19053187.

45. Ranc V, Srovnal J, Kvitek L, Hajduch M. Discrimination of circulating tumor cells of breast cancer and colorectal 
cancer from normal human mononuclear cells using Raman spectroscopy. Analyst. 2013;138(20):5983-8. DOI: 10.1039/c3an00855j PMID: 23945652.

46. Jenkins CA, Lewis PD, Dunstan PR, Harris DA. Role of Raman spectroscopy and surface enhanced Raman spectroscopy in colorectal cancer. World J Gastrointest Oncol. 2016;8(5):427-38. DOI: 10.4251/wjgo.v8.i5.427 PMID: 27190582.

47. Schie IW, Ruger J, Mondol AS, Ramoji A, Neugebauer U, Krafft C, et al. High-Throughput Screening Raman Spectroscopy Platform for Label-Free Cellomics. Anal Chem. 2018;90(3):2023-30. DOI: 10.1021/acs.analchem.7b04127 PMID: 29286634.

48. Shashni B, Horiguchi Y, Kurosu K, Furusho H, Nagasaki Y. Application of surface enhanced Raman spectroscopy as a diagnostic system for hypersialylated metastatic cancers. Biomaterials. 2017; 134:143-53. DOI: 10.1016/j.biomaterials.2017.04.038 PMID: 28460336.

49. Ito H, Hasegawa K, Hasegawa Y, Nishimaki T, Hosomichi K, Kimura S, et al. Silver Nanoscale Hexagonal Column Chips for Detecting Cell-free DNA and Circulating Nucleosomes in Cancer Patients. Sci Rep. 2015;5:10455. DOI: 10.1038/srep10455 PMID: 25994878.

50. Kelly JG, Najand GM, Martin FL. Characterisation of DNA methylation status using spectroscopy (mid-IR versus Raman) with multivariate analysis. J Biophotonics. 2011;4(5):345-54. DOI: 10.1002/jbio.201000085 PMID: 21520428.

51. Ouyang L, Hu Y, Zhu L, Cheng GJ, Irudayaraj J. A reusable laser wrapped graphene-Ag array based SERS sensor for trace detection of genomic DNA methylation. Biosens Bioelectron. 2017;92:755-62. DOI: 10.1016/j. bios.2016.09.072 PMID: 27825882.

52. Luo X, Jiang L, Kang T, Xing Y, Zheng E, Wu P, et al. Label-Free Raman Observation of TET1 Protein-Mediated Epigenetic Alterations in DNA. Anal Chem. 2019;91(11): 7304-12. DOI: 10.1021/acs.analchem.9b01004 PMID: 31074614.

53. Rohrig B, du Prel JB, Wachtlin D, Kwiecien R, Blettner M. Sample size calculation in clinical trials: part 13 of a series on evaluation of scientific publications. Dtsch Arztebl Int.
2010;107(31-32):552-6. DOI: 10.3238/arztebl.2010.0552 PMID: 20827353.

54. Kelly PJ, Webster AC, Craig JC. How many patients do we need for a clinical trial? Demystifying sample size calculations. Nephrology (Carlton). 2010;15(8):725-31. DOI: 10.1111/j.1440-1797.2010.01432.x PMID: 21175958.

55. Medical Advisory Secretariat. Fecal occult blood test for colorectal cancer screening: an evidence-based analysis. Ont Health Technol Assess Ser. 2009;9(10):1-40. PMID: 23074514.

56. Imperiale $\mathrm{TF}$, Ransohoff $\mathrm{DF}$, Itzkowitz $\mathrm{SH}$, Levin TR, Lavin P, Lidgard GP, et al. Multitarget stool DNA testing for colorectal-cancer screening. $\mathrm{N}$ Engl J Med. 2014;370(14):1287-97. DOI: 10.1056/NEJMoa1311194 PMID: 24645800.

57. Mandel JS, Bond JH, Church TR, Snover DC, Bradley GM, Schuman LM, et al. Reducing mortality from colorectal cancer by screening for fecal occult blood. Minnesota Colon Cancer Control Study. N Engl J Med. 1993;328(19):1365-71. DOI: 10.1056/NEJM199305133281901 PMID: 8474513.

58. Hardcastle JD, Chamberlain JO, Robinson MH, Moss SM, Amar SS, Balfour TW, et al. Randomised controlled trial of faecal-occult-blood screening for colorectal cancer. Lancet. 1996;348(9040):1472-7. DOI: 10.1016/S01406736(96)03386-7 PMID: 8942775.

59. Liu MC, Oxnard GR, Klein EA, Swanton C, Seiden MV, Liu MC, et al. Sensitive and specific multi-cancer detection and localization using methylation signatures in cell-free DNA. Ann Oncol. 2020;31(6):745-59. DOI: 10.1016/j.annonc.2020.02.011.

60. Timmermann B, Kerick M, Roehr C, Fischer A, Isau M, Boerno ST, et al. Somatic mutation profiles of MSI and MSS colorectal cancer identified by whole exome next generation sequencing and bioinformatics analysis. PLoS One. 2010;5(12):e15661. DOI: 10.1371/journal.pone.0015661 PMID: 21203531.

61. Winawer SJ, Zauber AG, Ho MN, O’Brien MJ, Gottlieb LS, Sternberg SS, et al. Prevention of colorectal cancer by colonoscopic polypectomy. The National Polyp Study Workgroup. N Engl J Med. 1993;329(27):1977-81. DOI: 10.1056/NEJM199312303292701 PMID: 8247072. 